\section{$\$$ Research Square \\ Preprints are preliminary reports that have not undergone peer review. They should not be considered conclusive, used to inform clinical practice, or referenced by the media as validated information.}

\title{
Epidemiology, Species Distribution, and Outcome of Nosocomial Candida spp. Bloodstream Infection in Shanghai - An 11-year Retrospective Analysis in a Tertiary Care Hospital
}

\author{
Yan-Jun ZHENG \\ Shanghai Jiao Tong University Medical School Affiliated Ruijin Hospital \\ Ting XIE \\ Suining Central Hospital \\ Lin WU \\ Shanghai Jiao Tong University Medical School Affiliated Ruijin Hospital \\ Xiao-Ying LIU \\ Shanghai Jiao Tong University Medical School Affiliated Ruijin Hospital \\ Ling ZHU \\ Shanghai Jiao Tong University Medical School Affiliated Ruijin Hospital \\ Ying CHEN \\ Shanghai Jiao Tong University Medical School Affiliated Ruijin Hospital

\section{En-Qiang MAO} \\ Shanghai Jiao Tong University Medical School Affiliated Ruijin Hospital \\ Li-Zhong HAN \\ Shanghai Jiao Tong University Medical School Affiliated Ruijin Hospital \\ Er-Zhen CHEN \\ Shanghai Jiao Tong University Medical School Affiliated Ruijin Hospital \\ Zhi-Tao Yang ( $\triangle$ yangzhitao@hotmail.fr) \\ Shanghai Jiao Tong University Medical School Affiliated Ruijin Hospital https://orcid.org/0000-0002-6289-6493
}

\section{Research Article}

Keywords: Candida spp., Bloodstream infection, Epidemiology, Species distribution, Antifungal therapy, Early treatment.

Posted Date: February 19th, 2021

DOI: https://doi.org/10.21203/rs.3.rs-212048/v1

License: (c) (i) This work is licensed under a Creative Commons Attribution 4.0 International License. Read Full License 


\section{Abstract}

\section{Background}

The incidence of Candida bloodstream infections (BSIs), has increased over time. In this study, we aimed to describe the current epidemiology of Candida BSI in a large tertiary care hospital in Shanghai and to determine the risk factors of 28-day mortality and the impact of antifungal therapy on clinical outcomes.

\section{Methods}

All consecutive adult inpatients with Candida BSI at Ruijin Hospital from 2008.1 to 2018.12 were enrolled. Underlying diseases, clinical severity, species distribution, antifungal therapy, and their impact on the outcomes were analyzed.

\section{Results}

Among the 370 inpatients with 393 consecutive episodes of Candida BSI, the incidence of nosocomial Candida BSI was 0.39 episodes/1000 hospitalized patients. Of the 393 cases, 299 (76.1\%) were treated with antifungal therapy (247 and 52 were treated with early appropriate and targeted antifungal therapy, respectively). The overall 28 -day mortality rate was $28.5 \%$, which was significantly lower in those who received early appropriate (25.5\%) or targeted (23.1\%) antifungal therapy than in those who did not (39.4\%; $\mathrm{P}=0.012$ and $\mathrm{P}=0.046$, respectively). In multivariate Cox regression analysis, age, chronic renal failure, mechanical ventilation, and neutropenia were found to be independent risk factors of 28-day mortality rate. Patients who received antifungal therapy had a lower mortality risk than did those who did not.

\section{Conclusions}

The incidence of Candida BSI has increased steadily in the past 11 years at our tertiary care hospital in Shanghai. Antifungal therapy influenced short-term survival, but no significant difference in mortality was observed between those who received early appropriate and targeted antifungal therapy.

\section{Background}

The incidence of invasive fungal infection has increased over time, especially for Candida bloodstream infections (BSIs), which is associated with considerable excess mortality and costs [1-3]. In the past two decades, the incidence of fungal infection has increased from 0.1 episodes/1000 admissions to $0.3-0.6$ episodes/1000 admissions in China, North America, and some European countries [4-7]. The mortality rate ranges from 35\%-53\% [8-11]. The optimal management of Candida BSIs included early awareness of patients at risk, control of the infection source, and timely administration of appropriate antifungal agents $[12,13]$.Consequently, antifungal agents have been widely used as empirical therapy. However, the overuse of antifungal agents results in increased costs, toxicity, and ecological selection pressure for antifungal resistance and adverse drug interactions. Several studies showed that delayed antifungal therapy (more than $48 \mathrm{~h}$ from onset) is associated with higher mortality [14], whereas others have reported conflicting results [15-17].

In this study, we retrospectively analyzed data from all patients with Candida BSI at our hospital between 2008 and 2018 , aiming to describe their clinical characteristics, species distribution, antifungal therapy and to determine the risk factors for 28-day mortality.

\section{Methods}

\subsection{Study setting and population}

A retrospective analysis of data on consecutive Candida BSI episodes in adult inpatients ( $\geq 18 y e a r s)$ between January 1, 2008 and December 31 , 2018, collected from the microbiology database of a 1900-bed teaching hospital in Shanghai, was performed.

Demographics and data on underlying diseases, comorbidities, severity of clinical features, Candida species distribution, and early appropriate or targeted antifungal treatment were compared among the patients with Candida BSI. Data on the initial and targeted antifungal agents used were also collected.

Candida BSI was defined as at least one positive blood culture for Candida. [18]. Neutropenia was defined as $<500 / \mathrm{mm}^{3}$ absolute neutrophil count. Prior corticosteroid was defined as receiving $>1 \mathrm{mg} / \mathrm{kg} / \mathrm{d}$ prednisone for more than one week or equivalent before Candida BSI onset.

\subsection{Laboratory methods}

Isolates were detected from blood cultures using the BACTEC ${ }^{T M}$ FX system (Becton Dickinson, Inc., Sparks, MD, USA), identified using matrix-assisted laser desorption ionization-time of flight mass spectrometer (bioMérieux, Marcy-l'Étoile, France). Susceptibility testing for flucytosine, amphotericin B, 
fluconazole, voriconazole, and itraconazole was performed using the ATB® FUNGUS 3 system (BioMérieux, France), which is widely used in China [19]. This test provides information on susceptibility to antifungals agents, which is concordant with that obtained using the methodologies of the Clinical and Laboratory Standards Institute (CLSI) and European Committee on Antimicrobial Susceptibility Testing (EUCAST) [20].

Early appropriate antifungal treatment was defined as commencement of appropriate drug treatment at an adequate dosage before obtaining in vitro susceptibility test results. The adequate dosage of the antifungal agent was defined according to 2009 or 2016 Infectious Diseases Society of America (IDSA) guidelines $[18,21]$. Targeted antifungal treatment was defined as commencement of appropriate targeted treatment after obtaining results from susceptibility testing, regardless of whether the antifungal treatment initiated was appropriate. Crude mortality was calculated from data on deaths registered 28 days after the occurrence of Candida BSI.

\subsection{Statistical analysis}

Descriptive and subgroup analyses were performed for the baseline characteristics, and continuous variables were expressed as mean \pm standard deviation (SD) or median and interquartile range according to their distributions. The chi-square test or 2-tailed Fisher exact test was applied to categorical variables. To identify the risk factors for 28-day mortality, multivariate Cox regression analysis was performed, and adjusted hazard ratio (HRs) with 95\% confidence intervals (Cls) were reported. Variables that were associated with 28-day mortality in the Cox univariate analyses with a $\mathrm{P}<0.05$ were entered into the multivariate Cox regression analysis model based on the forward selection. Two-tailed tests of significance at the level of a P value $<0.05$ level was considered as significant. Statistical analysis was performed using IBM SPSS Statistics for Windows, version 22.0 (IBM Corp., Armonk, N.Y. USA).

\subsection{Ethics}

The study was approved by Ruijin Hospital, Shanghai Jiao Tong University, School of Medicine institutional review board, and written informed consent was not required because of the observationalature of this study.

\section{Results}

\subsection{Incidence and clinical features of Candida BSI episodes}

Data on a total of 393 consecutive episodes of Candida BSI were collected from 370 inpatients during the 11-year study period. The demographic characteristics of the patients are summarized in Table 1. The mean age of the patients was $57.6 \pm 19.0$ years, and $74.3 \%$ were male. Candida BSI incidence was 0.39 episodes/1000 admissions. The incidence increased steadily from 0.21 (2008), to 0.59 (2017), to 0.33 episodes per 1,000 admissions (2018) (Figure 1[A]). Among the 393 Candida BSI episodes, 148 (37.7\%), 167 (42.5\%), and 78 (19.8\%) occurred in the surgical ward, intensive care units (ICUs), and internal medicine ward, respectively (Figure 1[B]).

C. albicans was isolated in $19.3 \%, 41.2 \%$ and $38.9 \%$, of cases in internal medicine wards, surgery wards, and the ICU, respectively (P=0.003). A higher proportion of $C$. tropicalis (34.7\%) was found in internal medicine wards than in the surgery wards (21.6\%) and ICUs (21.6\%).

Most patients with Candida BSI had at least one comorbidity. These included 118 (30\%) with solid tumors, 48 (12.2\%) had hematological malignancies, 77 (19.6\%) had diabetes mellitus, 124 (31.6\%) had chronic cardiac disease, 52 (13.2\%) had chronic pulmonary disease, $42(10.7 \%)$ had chronic renal failure, in 26 (6.6\%) patients, the skin barrier was considered compromised, 244 (62.1\%) had prior surgical intervention, 54 (13.7\%) used corticosteroid, 88 (22.4\%) used prior antifungal agents, and 255 (64.9\%) received antibiotics prior Candida BSI onset. A total of 244 (72\%) patients had at least two comorbidities. No patient had human immunodeficiency virus (HIV) infection. Regarding the severity, 309 (78.6\%) had fever, 180 (45.8\%) received parenteral nutrition, 147 (37.4\%) received mechanical ventilation, 49 (12.5\%) received renal replacement therapy, and 42 (10.7\%) had neutropenia. The clinical characteristics of patients by Candida species are shown in Table 1. 
Table 1

Demographic and clinical data for patients with Candida bloodstream infection

\begin{tabular}{|c|c|c|c|c|c|c|c|c|c|}
\hline & $\begin{array}{l}\text { C. } \\
\text { albicans }\end{array}$ & $\begin{array}{l}\text { C. } \\
\text { parapsilosis }\end{array}$ & $\begin{array}{l}\text { C. } \\
\text { tropicalis }\end{array}$ & $\begin{array}{l}\text { C. } \\
\text { glabrata }\end{array}$ & $\begin{array}{l}\text { C. } \\
\text { guilliermondii }\end{array}$ & $\begin{array}{l}\text { C. } \\
\text { sake }\end{array}$ & $\begin{array}{l}\text { C. } \\
\text { krusei }\end{array}$ & $\begin{array}{l}\text { Other } \\
\text { Candida } \\
\text { spp. }\end{array}$ & Total \\
\hline & $(n=141)$ & $(n=87)$ & $(n=69)$ & $(n=48)$ & $(n=20)$ & $(n=8)$ & $(n=5)$ & $(n=15)$ & $(n=393)$ \\
\hline Age, y & $65.2 \pm 14.5$ & $53.2 \pm 20.3$ & $50.5 \pm 19.5$ & $60.7 \pm 17.5$ & $50.5 \pm 19.4$ & $52 \pm 20.6$ & $40.8 \pm 27.1$ & $52.6 \pm 20.0$ & $57.6 \pm 19.0$ \\
\hline Male & 102(73.4) & $60(69.0)$ & 51(73.9) & 44(91.7) & 14(70) & $8(100)$ & $2(40)$ & 11(73.3) & 292(74.3) \\
\hline \multicolumn{10}{|l|}{ Origin } \\
\hline $\begin{array}{l}\text { Internal } \\
\text { medicine } \\
\text { ward }\end{array}$ & 15(10.6) & 19(21.8) & 27(39.1) & $7(14.6)$ & $3(15)$ & $0(0)$ & $3(60)$ & $4(26.7)$ & 78(19.8) \\
\hline Surgical ward & 61(43.3) & $32(36.8)$ & $16(23.2)$ & 15(31.3) & $10(50)$ & $6(75)$ & $0(0)$ & $8(53.3)$ & 148(37.7) \\
\hline ICU & $65(46.1)$ & $36(41.4)$ & $26(37.7)$ & $26(54.1)$ & $7(35)$ & $2(25)$ & $2(40)$ & $3(20)$ & $167(42.5)$ \\
\hline $\begin{array}{l}\text { Time from } \\
\text { admission to } \\
\text { infection, } d\end{array}$ & $30.6 \pm 35.3$ & $48.4 \pm 56.2$ & $37.7 \pm 32.4$ & $27.5 \pm 19.1$ & $35.9 \pm 52.5$ & $120.6 \pm 242.7$ & $64.2 \pm 68.6$ & $21.5 \pm 13.6$ & $37.6 \pm 53.1$ \\
\hline $\begin{array}{l}\text { Length of } \\
\text { hospital stay, } \\
\text { d }\end{array}$ & $56.0 \pm 54.9$ & $83.2 \pm 78.1$ & $71.5 \pm 56.1$ & $67.1 \pm 72.7$ & $97.7 \pm 142.6$ & $178.5 \pm 281.7$ & $92.4 \pm 88.5$ & $52.7 \pm 101.5$ & $71.1 \pm 82.8$ \\
\hline $\begin{array}{l}\text { Turnaround } \\
\text { Time, d }\end{array}$ & $4.3 \pm 1.9$ & $4.5 \pm 1.1$ & $3.8 \pm 1.1$ & $4.8 \pm 1.3$ & $4.3 \pm 1.2$ & $4.5 \pm 1.6$ & $4.4 \pm 1.5$ & $5.9 \pm 1.8$ & $4.4 \pm 1.5$ \\
\hline \multicolumn{10}{|l|}{$\begin{array}{l}\text { Underlying } \\
\text { disease }\end{array}$} \\
\hline Solid tumor & 47(33.3) & $26(29.9)$ & 13(18.8) & $17(35.4)$ & $6(30)$ & $2(25)$ & $0(0)$ & $7(46.7)$ & 118(30) \\
\hline $\begin{array}{l}\text { Hematologic } \\
\text { malignancy }\end{array}$ & $8(5.7)$ & $6(6.9)$ & $24(34.8)$ & $2(4.2)$ & $2(10)$ & $1(12.5)$ & $3(60)$ & 2(13.3) & $48(12.2)$ \\
\hline $\begin{array}{l}\text { Diabetes } \\
\text { mellitus }\end{array}$ & $33(23.4)$ & $21(24.1)$ & $8(11.6)$ & $9(18.8)$ & $1(5)$ & 1(12.5) & $0(0)$ & $4(26.7)$ & 77(19.6) \\
\hline $\begin{array}{l}\text { Chronic } \\
\text { cardiac } \\
\text { disease }\end{array}$ & $55(39)$ & $22(25.3)$ & 16(23.2) & 18(37.5) & $6(30)$ & $3(37.5)$ & $2(40)$ & $2(13.3)$ & 124(31.6) \\
\hline $\begin{array}{l}\text { Chronic } \\
\text { pulmonary } \\
\text { disease }\end{array}$ & $26(18.4)$ & $9(10.3)$ & $5(7.2)$ & $6(12.5)$ & $1(5)$ & $1(12.5)$ & $1(20)$ & $3(20)$ & $52(13.2)$ \\
\hline $\begin{array}{l}\text { Chronic } \\
\text { renal failure }\end{array}$ & 18(12.8) & $6(6.9)$ & $6(8.7)$ & $5(10.4)$ & $5(25)$ & $0(0)$ & $0(0)$ & $2(13.3)$ & 42(10.7) \\
\hline $\begin{array}{l}\text { Skin barrier } \\
\text { compromised }\end{array}$ & $5(3.5)$ & $9(10.3)$ & $5(7.2)$ & $1(2.1)$ & $3(15)$ & $3(37.5)$ & $0(0)$ & $0(0)$ & $26(6.6)$ \\
\hline $\begin{array}{l}\text { Prior } \\
\text { surgical } \\
\text { intervention } \\
\text { (<1 month) }\end{array}$ & $97(68.8)$ & $48(55.2)$ & $36(52.2)$ & $30(62.5)$ & $15(75)$ & $6(75)$ & $2(40)$ & $10(66.7)$ & $244(62.1)$ \\
\hline $\begin{array}{l}\text { Corticosteroid } \\
\text { use }\end{array}$ & $11(7.8)$ & $13(14.9)$ & 12(17.4) & $9(18.8)$ & $4(20)$ & $0(0)$ & $3(60)$ & $2(13.3)$ & $54(13.7)$ \\
\hline $\begin{array}{l}\text { Prior use of } \\
\text { antifungal } \\
\text { agents (<6 } \\
\text { months) }\end{array}$ & $20(14.2)$ & $19(21.8)$ & $24(34.8)$ & $11(22.9)$ & $8(40)$ & $1(12.5)$ & $4(80)$ & $1(6.7)$ & $88(22.4)$ \\
\hline \multicolumn{10}{|c|}{ Severity of clinical feature } \\
\hline $\begin{array}{l}\text { Fever } \\
\left(\mathrm{T}>38.2^{\circ} \mathrm{C}\right)\end{array}$ & 114(80.9) & $63(72.4)$ & $60(87)$ & $34(70.8)$ & $16(80)$ & $6(75)$ & $3(60)$ & 13(86.7) & $309(78.6)$ \\
\hline $\begin{array}{l}\text { Parenteral } \\
\text { nutrition }\end{array}$ & 71(50.4) & $42(48.3)$ & $28(40.6)$ & $23(47.9)$ & $5(25)$ & $3(37.5)$ & $2(40)$ & $6(40)$ & $180(45.8)$ \\
\hline $\begin{array}{l}\text { Mechanical } \\
\text { ventilation }\end{array}$ & $58(41.1)$ & $31(35.6)$ & 23(33.3) & $25(52.1)$ & $5(25)$ & $2(25)$ & $2(40)$ & $1(6.7)$ & $147(37.4)$ \\
\hline
\end{tabular}




\begin{tabular}{|c|c|c|c|c|c|c|c|c|c|}
\hline $\begin{array}{l}\text { Renal } \\
\text { replacement } \\
\text { therapy }\end{array}$ & 17(12.1) & 10(11.5) & $9(13)$ & $7(14.6)$ & $5(25)$ & $0(0)$ & $1(20)$ & $0(0)$ & $49(12.5)$ \\
\hline $\begin{array}{l}\text { Central } \\
\text { venous } \\
\text { catheter }\end{array}$ & $121(85.8)$ & $67(77)$ & 49(71) & $43(89.6)$ & $17(85)$ & $5(62.5)$ & $4(80)$ & $10(66.7)$ & $316(80.4)$ \\
\hline Neutropenia & $4(2.8)$ & $8(9.2)$ & $22(31.9)$ & $1(2.1)$ & $2(10)$ & $0(0)$ & $3(60)$ & 2(13.3) & $42(10.7)$ \\
\hline $\begin{array}{l}\text { 28-day } \\
\text { mortality }\end{array}$ & $54(38.3)$ & 16(18.4) & $19(27.5)$ & $13(27.1)$ & $3(15)$ & $1(12.5)$ & $1(20)$ & $5(33.3)$ & $112(28.5)$ \\
\hline \multicolumn{10}{|c|}{$\begin{array}{l}\text { Other Candida spp. Includes C. gum (4 cases), C. Iusitaniae (3 cases), C. intermedia (2 cases), C. lipolytica (2cases), C. theae (2 cases), C.famata } \\
\text { (1 case), and C. haemulonii (1 case). }\end{array}$} \\
\hline
\end{tabular}

\subsection{Antifungal susceptibility of Candida isolates}

A total of 393 Candida spp. were isolated, including 141 (35.9\%), C. albicans, 87, C. parapsilosis (22.1\%); 69, C. tropicalis (17.6\%); 48, C. glabrata (12.2\%); 20, C. guilliermondii (5.1\%); 8, C. sake (2.0\%); 5, C.krusei (1.3\%); and 15, other species (4, C. gum; 3, C. lusitaniae; 2 , C. intermedia; 2, C. theae; 2, C. lipolytica; 1, C. famata; and 1, C. haemulonii).

Among the 393 Candida species, 378 were subjected to antifungal susceptibility testing, on the basis of 2012 CLSI breakpoints (CBPs). As shown in Table 2, the susceptibility of $C$. albicans, $C$. parapsilosis to fluconazole and voriconazole were quite high, compared to that to itraconazole ( $94 \%$, $93.3 \%$ vs. $82.1 \%)$. The susceptibility of $C$. tropicalis to triazoles fluconazole, voriconazole, and itraconazole was unsatisfactory. Amphotericin B and 5-flucytosine remained superior against common Candida spp., except for C. krusei and C. guilliermondii, with 95\% susceptibility. 
Table 2

Antifungal susceptibility testing results (ATB Fungus 3 ) of 378 Candida [n (\%)]

\begin{tabular}{|c|c|c|c|c|c|c|c|c|c|}
\hline & $\begin{array}{l}\text { C.albicans } \\
(\mathrm{n}=134)\end{array}$ & $\begin{array}{l}\text { C.parapsilosis } \\
(\mathrm{n}=86)\end{array}$ & $\begin{array}{l}\text { C.tropicalis } \\
(n=67)\end{array}$ & $\begin{array}{l}\text { C.glabrata } \\
(n=47)\end{array}$ & $\begin{array}{l}\text { C.krusei } \\
(n=5)\end{array}$ & $\begin{array}{l}\text { C.sake } \\
(\mathrm{n}=8)\end{array}$ & $\begin{array}{l}\text { C.guilliermondii } \\
(\mathrm{n}=19)\end{array}$ & $\begin{array}{l}\text { Other } \\
\text { Candida } \\
\text { spp. }(\mathrm{n}=12)\end{array}$ & $\begin{array}{l}\text { Total } \\
\llbracket n=378 \rrbracket\end{array}$ \\
\hline \multicolumn{10}{|c|}{ Fluconazole } \\
\hline S & $126(94)$ & $77(89.5)$ & 35 (52.2) & $0(0)$ & $0(0)$ & $8(100)$ & $13(68.4)$ & $9(75.0)$ & $\begin{array}{l}268 \\
\square 70.9 \square\end{array}$ \\
\hline SDD & $1(0.8)$ & $6(7.0)$ & $3(4.5)$ & $44(93.6)$ & $0(0)$ & $0(0)$ & $0(0)$ & $0(0)$ & $\begin{array}{l}54 \\
\square 14.3 \rrbracket\end{array}$ \\
\hline $\mathbf{R}$ & $7(5.2)$ & $3(3.5)$ & 29 (43.3) & $3(6.4)$ & $5(100)$ & $0(0)$ & $6(31.6)$ & $3(25.0)$ & 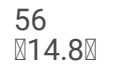 \\
\hline \multicolumn{10}{|c|}{ Itraconazole } \\
\hline S & $110(82.1)$ & 75 (87.2) & 25 (37.3) & $0(0)$ & $0(0)$ & $8(100)$ & $6(31.6)$ & $9(75.0)$ & 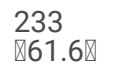 \\
\hline SDD & $6(4.5)$ & 7 (8.1区 & $4(6.0)$ & $40(85.1)$ & $2(40.0)$ & $0(0)$ & $7(36.8)$ & $0(0)$ & $\begin{array}{l}66 \\
\otimes 17.5 \square\end{array}$ \\
\hline $\mathbf{R}$ & $18(13.4)$ & $4 \otimes 4.7 \rrbracket$ & 38 (56.7) & 7 (14.9) & $3(60.0)$ & $0(0)$ & $6(31.6)$ & $3(25.0)$ & 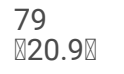 \\
\hline \multicolumn{10}{|c|}{ Voriconazole } \\
\hline$S$ & 125 (93.3) & 79 (91.9) & $41(61.2)$ & $45(95.8)$ & $4(80.0)$ & $8(100)$ & $12(63.2)$ & 11 (91.7) & $\begin{array}{l}325 \\
\square 86.0 \bigotimes\end{array}$ \\
\hline SDD & $0(0)$ & $2(2.3)$ & $2(3.0)$ & $1(2.1)$ & $1(2.0)$ & $0(0)$ & $3(15.8)$ & $0(0)$ & $9 \llbracket 2.4 \rrbracket$ \\
\hline $\mathbf{R}$ & $9(6.7)$ & $5(5.8)$ & $24(35.8)$ & $1(2.1)$ & $0(0)$ & $0(0)$ & $4(21.0)$ & $1(8.3)$ & 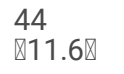 \\
\hline \multicolumn{10}{|c|}{$\begin{array}{l}\text { Amphotericin } \\
\text { B }\end{array}$} \\
\hline$S$ & 133 (99.3) & $83(96.5)$ & $67(100)$ & $47(100)$ & $5(100)$ & $8(100)$ & $18(94.7)$ & 11 (91.7) & 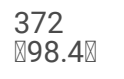 \\
\hline $\mathbf{R}$ & $1(0.7)$ & $3(3.5)$ & $0(0)$ & $0(0)$ & $0(0)$ & $0(0)$ & $1(5.3)$ & $1(8.3)$ & $6 \otimes 1.6 \rrbracket$ \\
\hline \multicolumn{10}{|c|}{ Flucytosine } \\
\hline S & 132 (98.5) & 85 (98.8) & 65 (97.0) & 46 (97.9) & $1(20.0)$ & $8(100)$ & $9(47.4)$ & $12(100)$ & 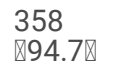 \\
\hline $\mathbf{R}$ & $2(1.5)$ & $1(1.2)$ & $2(3.0)$ & $1(2.1)$ & $4(80.0)$ & $0(0)$ & $10(52.6 \rrbracket$ & $0(0)$ & $20 \otimes 5.3 \rrbracket$ \\
\hline
\end{tabular}

15 Candida spp. isolates did not have a susceptibility test, C. albicans (7), C. parapsilosis (2), C. tropicalis (2), and glabrata, theae, gum, haemulonii each.

R, resistance; S, susceptible; SDD, susceptible dose dependence.

\subsection{Antifungal therapy and outcome of patients with Candida BSI}

Antifungal therapy was administered to 299 (76.1\%) patients, whereas 94 (23.9\%) patients did not receive antifungal therapy. Among those who received antifungal therapy, $247(62.8 \%)$ received early appropriate antifungal therapy, and $52(13.2 \%)$ received targeted antifungal therapy. Fluconazole was most frequently used as an empirical therapy, followed by echinocandins and voriconazole. Eighteen (4.6\%) patients with Candida BSI received combination therapy.

The overall, 28-day mortality rate was $28.5 \%$, and the rate was significantly higher in internal medicine wards and ICUs than in surgical wards ( $37.2 \%$ and $34.7 \%$ vs. $16.9 \%$, respectively, $\mathrm{P}<0.001)$. The mortality rates among those who received early appropriate or targeted antifungal therapy was $26.8 \%$ and $25.1 \%(P=0.012$ or $P=0.046)$, compared to $39.3 \%$ for those who did not receive any antifungal therapy, with no significant difference between those who received early appropriate antifungal therapy and those who received targeted antifungal therapy. 
On univariate analysis, age, solid tumor, diabetes mellitus, chronic cardiac disease, chronic renal failure, skin disease, prior surgical intervention, mechanical ventilation, neutropenia, and antifungal therapy were found to be associated with 28-day mortality. On multivariate Cox regression analysis, advanced age $(\mathrm{HR}=1.025 ; 95 \% \mathrm{Cl}, 1.013-1.037 ; \mathrm{P}<0.001)$, chronic renal failure $(\mathrm{HR}=2.018 ; 95 \% \mathrm{Cl} 1.234-3.299 ; \mathrm{P}=0.005)$, mechanical ventilation $(\mathrm{HR}=1.950 ; 95 \% \mathrm{Cl} 1.307-2.912 ; \mathrm{P}=0.001)$, and neutropenia $(\mathrm{HR}=4.347 ; 95 \% \mathrm{Cl} 2.462-7.675 ; \mathrm{P}<0.001)$, were found to be independent risk factors for 28-day mortality. However, antifungal therapy ( $\mathrm{HR}=0.570 ; 95 \% \mathrm{Cl} 0.382-0.849 ; \mathrm{P}=0.006)$ was an independent protective factor for $28-\mathrm{day}$ mortality (Table 3).

\begin{tabular}{|c|c|c|c|c|c|}
\hline \multicolumn{6}{|c|}{ Table 3} \\
\hline \multicolumn{3}{|c|}{ 28-day outcome } & \multicolumn{3}{|c|}{ Multivariable analysis } \\
\hline & Survival ( $n=281)$ & Death $(n=112)$ & P-value & $\mathrm{HR}(95 \% \mathrm{Cl})$ & P-value \\
\hline Male & $216(76.9)$ & $76(67.9)$ & 0.065 & - & - \\
\hline Age, y & $55.2(19.5)$ & $63.6(16.2)$ & $<0.01$ & $1.025(1.013-1.037)$ & $<0.001$ \\
\hline Underlying disease & & & & - & - \\
\hline Solid tumor & $91(32.4)$ & $27(24.1)$ & 0.106 & - & - \\
\hline Hematologic malignancy & $32(11.4)$ & $16(14.3)$ & 0.428 & - & - \\
\hline Diabetes mellitus & $49(17.4)$ & $28(25)$ & 0.088 & - & - \\
\hline Chronic Cardiac disease & $72(25.6)$ & $52(46.4)$ & $<0.01$ & - & 0.105 \\
\hline Chronic Pulmonary disease & $34(12.1)$ & $18(16.1)$ & 0.294 & - & - \\
\hline Chronic renal failure & $20(7.1)$ & 22(19.6) & $<0.01$ & $2.018(1.234-3.299)$ & 0.005 \\
\hline Skin barrier compromised & $24(8.5)$ & $2(1.8)$ & 0.015 & - & 0.308 \\
\hline Prior surgical intervention (<1month) & $182(64.8)$ & $62(55.4)$ & 0.083 & - & - \\
\hline Corticosteroid use & $40(14.2)$ & $14(12.5)$ & 0.652 & - & - \\
\hline Prior antifungal agents use (<6month) & $64(22.8)$ & $24(21.4)$ & 0.772 & - & - \\
\hline Severity of clinical feature & & & & - & - \\
\hline Fever $\left(\mathrm{T}>38.2^{\circ} \mathrm{C}\right)$ & $220(78.3)$ & $89(79.5)$ & 0.798 & - & - \\
\hline Parenteral nutrition & $124(44.1)$ & $56(50)$ & 0.292 & - & - \\
\hline Mechanical ventilation & $89(31.7)$ & $58(51.8)$ & $<0.01$ & $1.950(1.307-2.912)$ & 0.001 \\
\hline Renal replacement therapy & $32(11.4)$ & $17(15.2)$ & 0.305 & - & - \\
\hline Central venous catheter & $227(80.8)$ & $89(79.5)$ & 0.766 & - & - \\
\hline Neutropenia & $24(8.5)$ & $18(16.1)$ & 0.029 & $4.347(2.462-7.675)$ & $<0.001$ \\
\hline Antifungal therapy & $224(74.9)$ & $75(25.1)$ & \multirow[t]{2}{*}{0.007} & \multirow[t]{2}{*}{$0.502(0.294-0.857)$} & \multirow[t]{2}{*}{0.006} \\
\hline No treatment & $57(60.6)$ & $37(39.4)$ & & & \\
\hline
\end{tabular}

\section{Discussion}

Our study showed that the incidence of Candida BSI has increased steadily in the past 11 years at our tertiary care hospital in Shanghai. Several studies have shown a substantial increase in Candida BSI incidence in the past two decades, which is similar to our study findings [4, 11, 22]. Intensive use of broad-spectrum antibiotics may be the main cause. In addition, gradually worsening hospitalized patient profiles, underlying comorbidities including malignancy, and a high frequency of surgeries may be predisposing risk factors for increased incidence.

C. albicans remains the most common pathogen causing Candida BSI. However, over the past two decades, an increased percentage of common non-C. albicans Candida spp. have been reported worldwide. In our study, non-C. albicans accounted for 64.1\%. C. parapsilosis, C. tropicalis and C. 
glabrata accounted for most of the non-C. albicans species. However, some studies in western countries showed that C. glabrata was the most frequent species among non-C. albicans, whereas C. tropicalis was quite rare compared to the incidence reported in our study (5.9\% and $7.0 \%$ vs. $27.4 \%)[23,24]$. In this study, the incidence of C. guilliermondii was significantly higher than that in other studies (4.8\% vs. $0.4 \%)[25]$. This could be related to the higher rate of parenteral nutrition and surgery (45.8\% and $62.1 \%)$ in the current study, as some research implied intravenous nutrition and surgery as being significant risk factors for Candida BSI due to C. guilliermondii. [26].

The antifungal susceptibility testing showed that the susceptibility of C. albicans, C. parapsilosis and C. sake to fluconazole were quite high (94\%, $89.5 \%, 100 \%)$. On the other hand, the resistance rate of $\mathrm{C}$. tropicalis for fluconazole was as high as $43.3 \%$, which was significantly higher than those reported in other studies abroad $[27,28]$. This may deserve more attention from clinicians in actual application.

The contributing factors of the finding of quite low 28-day mortality rate for Candida BSI in patients from surgical wards compared to those of other wards need further study.

Since there was no significant difference in the 28-day mortality rate between the patients who received early appropriate (26.8\%) or targeted antifungal therapy (25.1\%), we further analyzed the demographic data, underlying diseases, and clinical features between these two groups. We found no significant differences between the two groups in age $(p=0.33)$, sex $(p=0.89)$, number of underlying diseases ( $p=0.32)$, and number of severe clinical features $(p=0.96)$. The choice of antifungal agents between these two groups was further analyzed. In the early appropriate antifungal therapy group, the rate of azoles use was $50.6 \%$, and the rate of echinocandin use was $30.3 \%$. In the targeted antifungal therapy group, the rate of azoles use was $65.4 \%$, and the rate of echinocandin use was $17.3 \%$. The rate of echinocandin use was lower in the targeted antifungal therapy group than in the early appropriate antifungal therapy group $(p=0.038)$. Is the lower rate of echinocandin use in the targeted antifungal therapy group one of the reasons that caused no significant difference in mortality between the two groups? Many studies have confirmed the important role of echinocandin in antifungal therapy. Echinocandin has been recommended as a first-line antifungal agent since 2009[18], with fluconazole as an acceptable alternative for selected patients, reflecting the efficacy demonstrated by echinocandins and increasing resistance observed with fluconazole[29, 30]. Therefore, we believe that lower rate of echinocandin use in the delayed antifungal treatment group is unlikely to lead to a decrease in the 28-day mortality rate.

On the basis the above analysis, we suggest that early antifungal therapy has no significant impact on the 28-day mortality rate compared with targeted antifungal therapy. Many clinicians currently administer empirical antifungal agents, and our study results could be used to guide the clinical care of their patients. Overuse of antifungal drugs inevitably leads to a waste of medical resources and increased drug resistance[31]. If empirical antifungal agents cannot improve mortality, we should reconsider their use. Several studies support our findings[15-17] that the severity of illness (APACHE-II Score) affected short-term survival in patients with Candida infection, whereas the choice of initial antifungal agents did not affect short-term survival. Trifi et al. revealed no beneficial impact of an empirical antifungal therapy on 28- day survival or in preventing the occurrence of candidemia in critically ill patients with non-neutropenic sepsis [32]. However, some studies differed in their findings on the timing of antifungal agents' use. Bassetti et al. showed that the use of antifungal agents within 48 hours of obtaining a positive blood culture result is an independent protective factor against mortality during hospitalization[14]; Similarly, Tedeschi et al. showed that the administration of appropriate antifungal agents within 72 hours after blood culture is a protective factor for mortality during hospitalization[33]. However, our results revealed similar risk factors (age, neutropenia, and mechanical ventilation) and protective factors (antifungal therapy) for 28-day mortality.

Although our data were collected from a large hospital in Shanghai, several limitations of this study should be taken into consideration. First, this was a retrospective study performed at a single center, which could lead to selection bias. Moreover, the severity of illness score (such as APACHE II score) and the timing of CVC removal were not included for analysis because of missing data. Further, because echinocandin susceptibility testing has not been carried out at our hospital, relevant clinical data could not be obtained.

\section{Conclusions}

Our retrospective study findings showed increased incidence of Candida BSI in the past 11 years in Shanghai. Although the percentage of non- C. albicans spp. has been increasing, C. albicans spp. remains the most frequently isolated species. The mortality of patients with Candida BSI was quite high, especially in internal medicine wards. Antifungal therapy improved the short-term survival of patients with Candida BSI. Whether preemptive antifungal therapy should be initiated or antifungal therapy should be initiated after the antifungal susceptibility test needs further discussion.

\section{Abbreviations}

BSI, bloodstream infection; Cl, confidence interval; CLSI, Clinical and Laboratory Standards Institute; EUCAST, European Committee on Antimicrobial Susceptibility Testing; HR, hazard ratio; HIV, human immunodeficiency virus; ICU, intensive care unit; IDSA, Infectious Diseases Society of America; $\mathrm{SD}$, standard deviation

\section{Declarations}




\section{Funding:}

This work was supported by the Program for Outstanding Medical Academic Leader, National Nature Science Foundation of China [81772107,81772040], Scientific and Technological Innovation Act Program of Science and Technology Commission of Shanghai Municipality [18411950900], Key Discipline Construction Project of Shanghai Municipal Commission of Health and Family Planning [2016ZB0206], Clinical Research Innovation Project of Shanghai Hospital Development Center [SHDC12017116] and Program of Shanghai Jiao Tong University School of Medicine [DLY201803].

\section{Competing Interests:}

The authors declare that they have no competing interests.

\section{Availability of data and materials:}

All data generated or analyzed during this study are included in this published article.

\section{Code availability:}

Not applicable

\section{Authors' contributions:}

ZTY, EZC, and EQM made substantial contributions to conception and design. ZTY, LZH,YJZ, LW, TX LZ, and XYL participated in the acquisition of data. ZTY, YJZ, and YC drafted the manuscript. ZTY and EZC revised it critically.

\section{Ethics Approval:}

The study was approved by the local institutional review board (Ruijin Hospital, Shanghai Jiao Tong University, School of medicine).

\section{Consent to participate:}

Not applicable

\section{Consent for publication:}

Not applicable

\section{Acknowledgments:}

We thank Ms. Wen-Jing ZHENG to produce the massive time first to the article English grammar, the usage, and so on has carried on the revision.

\section{References}

1. Gudlaugsson O, Gillespie S, Lee K, Vande Berg J, Hu J, Messer S, Herwaldt L, Pfaller M, Diekema D (2003) Attributable mortality of nosocomial candidemia, revisited. Clinical infectious diseases: an official publication of the Infectious Diseases Society of America 37(9):1172-1177

2. Zaoutis TE, Argon J, Chu J, Berlin JA, Walsh TJ, Feudtner C (2005) The epidemiology and attributable outcomes of candidemia in adults and children hospitalized in the United States: a propensity analysis. Clinical infectious diseases: an official publication of the Infectious Diseases Society of America 41(9):1232-1239

3. Falagas ME, Apostolou KE, Pappas VD (2006) Attributable mortality of candidemia: a systematic review of matched cohort and case-control studies. European journal of clinical microbiology infectious diseases: official publication of the European Society of Clinical Microbiology 25(7):419-425

4. Bitar D, Lortholary O, Le Strat Y, Nicolau J, Coignard B, Tattevin P, Che D, Dromer F (2014) Population-based analysis of invasive fungal infections, France, 2001-2010. Emerg Infect Dis 20(7):1149-1155 
5. Jia X, Li C, Cao J, Wu X, Zhang L (2018) Clinical characteristics and predictors of mortality in patients with candidemia: a six-year retrospective study. European Journal of Clinical Microbiology Infectious Diseases 37(9):1717-1724

6. Marchetti O, Bille J, Fluckiger U, Eggimann P, Ruef C, Garbino J, Calandra T, Glauser M-P, Täuber MG, Pittet D, Switzerland FINo (2004) Epidemiology of Candidemia in Swiss Tertiary Care Hospitals: Secular Trends, 1991-2000. Clin Infect Dis 38(3):311-320

7. Wisplinghoff H, Bischoff T, Tallent SM, Seifert H, Wenzel RP, Edmond MB (2004) Nosocomial Bloodstream Infections in US Hospitals: Analysis of 24,179 Cases from a Prospective Nationwide Surveillance Study. Clin Infect Dis 39(3):309-317

8. Horn DL, Neofytos D, Anaissie EJ, Fishman JA, Steinbach WJ, Olyaei AJ, Marr KA, Pfaller MA, Chang CH, Webster KM (2009) Epidemiology and outcomes of candidemia in 2019 patients: data from the prospective antifungal therapy alliance registry. Clinical infectious diseases: an official publication of the Infectious Diseases Society of America 48(12):1695-1703

9. Wisplinghoff H, Ebbers J, Geurtz L, Stefanik D, Major Y, Edmond MB, Wenzel RP, Seifert H (2014) Nosocomial bloodstream infections due to Candida spp. in the USA: species distribution, clinical features and antifungal susceptibilities. Int J Antimicrob Agents 43(1):78-81

10. Kim SH, Yoon YK, Kim MJ, Sohn JW (2013) Clinical impact of time to positivity for Candida species on mortality in patients with candidaemia. J Antimicrob Chemother 68(12):2890-2897

11. Tiraboschi IN, Pozzi NC, Farias L, Garcia S, Fernandez NB (2017) [Epidemiology, species, antifungal resistance and outcome of candidemia in a university hospital in Buenos Aires, Argentina for 16 years]. Revista chilena de infectologia: organo oficial de la Sociedad Chilena de Infectologia 34 (5):431-440

12. Bassetti M, Peghin M, Timsit JF (2016) The current treatment landscape: candidiasis. J Antimicrob Chemother 71(suppl 2):ii13-ii22

13. Murri R, Giovannenze F, Camici M, Torelli R, Ventura G, Scoppettuolo G, Sanguinetti M, Cauda R, Fantoni M (2018) Systematic clinical management of patients with candidemia improves survival. J Infect 77(2):145-150

14. Bassetti M, Molinari MP, Mussap M, Viscoli C, Righi E (2013) Candidaemia in internal medicine departments: the burden of a rising problem. Clin Microbiol Infect 19(6):E281-E284

15. Parkins MD, Sabuda DM, Elsayed S, Laupland KB (2007) Adequacy of empirical antifungal therapy and effect on outcome among patients with invasive Candida species infections. J Antimicrob Chemother 60(3):613-618

16. Taur Y, Cohen N, Dubnow S, Paskovaty A, Seo SK (2010) Effect of antifungal therapy timing on mortality in cancer patients with candidemia. Antimicrob Agents Chemother 54(1):184-190

17. Grim SA, Berger K, Teng C, Gupta S, Layden JE, Janda WM, Clark NM (2011) Timing of susceptibility-based antifungal drug administration in patients with Candida bloodstream infection: correlation with outcomes. J Antimicrob Chemother 67(3):707-714

18. Pappas PG, Kauffman CA, Andes D, Benjamin DK Jr, Calandra TF, Edwards JE Jr, Filler SG, Fisher JF, Kullberg B-J, Zeichner LO, Reboli AC, Rex JH, Walsh TJ, Sobe JD (2009) Clinical Practice Guidelines for the Management Candidiasis: 2009 Update by the Infectious Diseases Society of America. Clin Infect Dis 48(5):503-535

19. Li F, Wu L, Cao B, Zhang Y, Li X, Liu Y (2013) Surveillance of the prevalence, antibiotic susceptibility, and genotypic characterization of invasive candidiasis in a teaching hospital in China between 2006 to 2011. BMC Infect Dis 13:353

20. Zhang L, Wang H, Xiao M, Kudinha T, Mao LL, Zhao HR, Kong F, Xu YC (2014) The widely used ATB FUNGUS 3 automated readings in China and its misleading high MICs of Candida spp. to azoles: challenges for developing countries' clinical microbiology labs. PloS one 9(12):e114004

21. Pappas PG, Kauffman CA, Andes DR, Clancy CJ, Marr KA, Ostrosky-Zeichner L, Reboli AC, Schuster MG, Vazquez JA, Walsh TJ, Zaoutis TE, Sobel JD (2016) Clinical Practice Guideline for the Management of Candidiasis: 2016 Update by the Infectious Diseases Society of America. Clinical infectious diseases: an official publication of the Infectious Diseases Society of America 62(4):e1-e50

22. Ulu Kilic A, Alp E, Cevahir F, Ture Z, Yozgat N (2017) Epidemiology and cost implications of candidemia, a 6-year analysis from a developing country. Mycoses 60(3):198-203

23. Schroeder M, Weber T, Denker T, Winterland S, Wichmann D, Rohde H, Ozga A-K, Fischer M, Kluge S (2020) Epidemiology, clinical characteristics, and outcome of candidemia in critically ill patients in Germany: a single-center retrospective 10-year analysis. Annals of Intensive Care 10 (1)

24. Ricotta EE, Lai YL, Babiker A, Strich JR, Kadri SS, Lionakis MS, Prevots DR, Adjemian J Invasive candidiasis species distribution and trends, United States, 2009-2017. LID - jiaa502 [pii] LID - 10.1093/infdis/jiaa502 [doi]. (1537-6613 (Electronic))

25. Pfaller MA, Andes DR, Diekema DJ, Horn DL, Reboli AC, Rotstein C, Franks B, Azie NE (2014) Epidemiology and outcomes of invasive candidiasis due to non-albicans species of Candida in 2,496 patients: data from the Prospective Antifungal Therapy (PATH) registry 2004-2008. PloS one 9 (7):e101510

26. Wu Z, Liu Y, Feng X, Liu Y, Wang S, Zhu X, Chen Q, Pan S (2014) Candidemia: incidence rates, type of species, and risk factors at a tertiary care academic hospital in China. Int $\mathrm{J}$ Infect Dis 22:4-8

27. Maria S, Barnwal G, Kumar A, Mohan K, Vinod V, Varghese A, Biswas R (2018) Species distribution and antifungal susceptibility among clinical isolates of Candida parapsilosis complex from India. Revista Iberoamericana de Micología 35(3):147-150

28. Neji S, Hadrich I, Trabelsi H, Abbes S, Cheikhrouhou F, Sellami H, Makni F, Ayadi A (2017) Virulence factors, antifungal susceptibility and molecular mechanisms of azole resistance among Candida parapsilosis complex isolates recovered from clinical specimens. Journal of biomedical science 24(1):67 
29. Kett DH, Shorr AF, Reboli AC, Reisman AL, Biswas P, Schlamm HT (2011) Anidulafungin compared with fluconazole in severely ill patients with candidemia and other forms of invasive candidiasis: support for the 2009 IDSA treatment guidelines for candidiasis. Crit Care (London England) 15(5):R253

30. Cui N, Wang H, Qiu H, Li R, Liu D (2017) Impact of initial empirical antifungal agents on the outcome of critically ill patients with invasive candidiasis: analysis of the China-SCAN study. Int J Antimicrob Agents 50(1):74-80

31. Hamdy RF, Zaoutis TE, Seo SK (2017) Antifungal stewardship considerations for adults and pediatrics. Virulence 8(6):658-672

32. Trifı A, Abdellatif S, Daly F, Nasri R, Touil Y, Ben Lakhal S (2019) Empiric antifungal and outcome in ICU patients. La Tunisie medicale 97(4):579587

33. Tedeschi S, Tumietto F, Giannella M, Bartoletti M, Cristini F, Cioni G, Ambretti S, Carretto E, Sambri V, Sarti M, Viale P (2016) Epidemiology and outcome of candidemia in internal medicine wards: A regional study in Italy. Eur J Intern Med 34:39-44

Figures

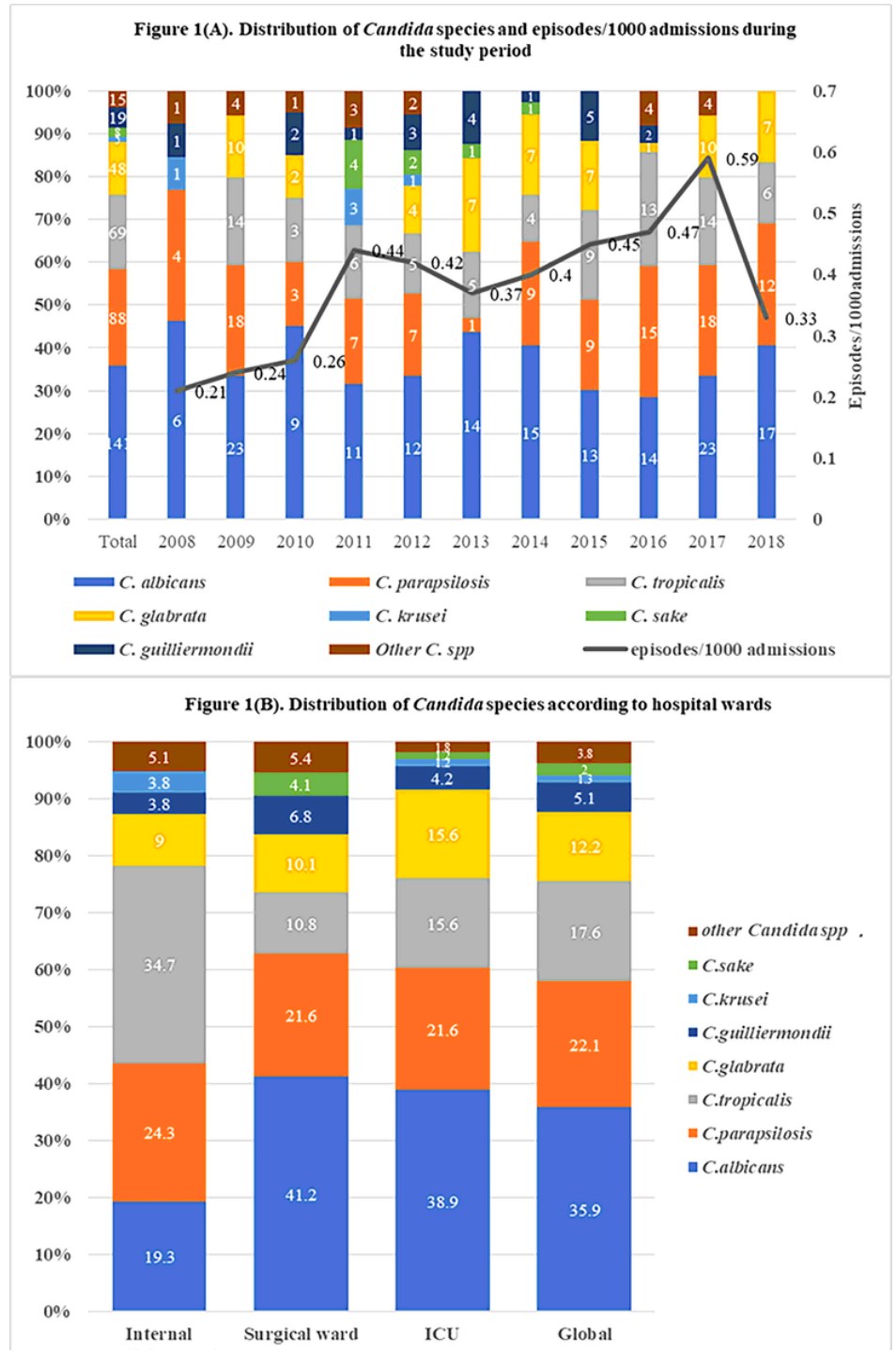

Figure 1

A) Distribution of Candida spp. and episodes/1000 admissions during the study period. Fig.1(B) Distribution of Candida spp. according to hospital wards;148 episodes were from surgical wards, 167 episodes were from ICUs and 78 episodes were from the internal medicine wards. 